\title{
MINIREVIEW
}

\section{Regulation of metabolism in Caenorhabditis elegans longevity}

\author{
Marco Gallo and Donald L Riddle*,2 \\ See research article http://www.biomedcentral.com/1741-7007/8/14
}

\begin{abstract}
The nematode Caenorhabditis elegans is a favorite model for the study of aging. A wealth of genetic and genomic studies show that metabolic regulation is a hallmark of life-span modulation. A recent study in BMC Biology identifying metabolic signatures for longevity suggests that amino-acid pools may be important in longevity.
\end{abstract}

Aging in Caenorhabditis elegans seems to be intimately tied to the modulation of metabolism. The first genes identified for their ability to affect life span were age-1, which encodes a phosphatidylinositol kinase [1], and daf-2 (constitutive dauer formation), which encodes a receptor tyrosine kinase similar to the mammalian insulin and insulin-like growth factor 1 (IGF1) receptors [2]. Although complete loss of daf-2 function is lethal [3], partial loss of function extends adult life by two- to threefold. daf-2 and age-1 specify steps in the insulin/ insulin-like signaling (IIS) pathway, thereby influencing metabolism. IIS signaling inactivates the FOXO (forkhead box O) transcription factor DAF-16, which controls genes that mediate a wide variety of functions, including metabolism, innate immunity, stress response, and translation (reviewed in [4]). The modulation of life span by the IIS pathway seems to result from an intricate network of physiological changes primarily mediated by unregulated DAF-16 activity, including major shifts in both energy and fat metabolism.

In addition to the IIS pathway, two other main groups of genes have profound effects on life span and are linked to metabolic control: genes involved in dietary restriction

\footnotetext{
${ }^{*}$ Correspondence: driddle@msl.ubc.ca

'Department of Medical Genetics, Faculty of Medicine, 263-2185 East Mall, The University of British Columbia, Vancouver, BC, V6T 1 Z4 Canada

${ }^{2}$ Michael Smith Laboratories, The University of British Columbia, Vancouver, BC, V6T 1 Z4 Canada
}

and mitochondrial function. Dietary restriction has been associated with increased life span in a wide variety of species (including mammals) and appears to be universally associated with longevity (reviewed in [5]). Increased longevity resulting from mutations in genes that regulate mitochondrial function is more problematic, although, at least superficially, it is related to metabolic slow-down. Work in nematodes and other invertebrates seems to support the notion that reducing the rate of oxidative phosphorylation (OXPHOS) leads to increased life span in two major ways: by decreasing the metabolic rate, and by reducing the level of reactive oxygen species, which are an obligate byproduct of OXPHOS. However, mutations that reduce mitochondrial function are beneficial for $C$. elegans longevity, but have deleterious and often lethal effects in mammals [6]. The reason for this discrepancy is not understood.

Given the large number of genes that have been shown to regulate life span through different pathways, it is reasonable to ask whether there are any physiological changes conducive to longevity that are common in all long-lived mutants. A recent metabolomic study on five different long-lived mutants published in BMC Biology by Fuchs et al. [7] suggests that the answer to this question may be yes. Fuchs et al. report that mutants carrying three different alleles of $d a f-2$, one allele of the insulinlike gene $d a f-28$ or one allele of ife-2 (translational initiation factor $4 \mathrm{E}$ ) have similar metabolic signatures, which cluster away from those of wild-type worms. These metabolic signatures are consistent with previous findings that assessed gene expression in daf-2 mutants [8]. In particular, they showed that long-lived daf-2 worms upregulate the glyoxylate cycle, gluconeogenesis and starch metabolism.

Fuchs et al. [7] detected a common metabolic signature for IIS mutants and ief-2. All five of the long-lived mutants had increased pools of amino acids, especially of the branched-chain amino acids isoleucine, leucine and valine, together with phenylalanine and tyrosine. A similar increase in branched-chain amino acids has been found in animals carrying mutations in components of 


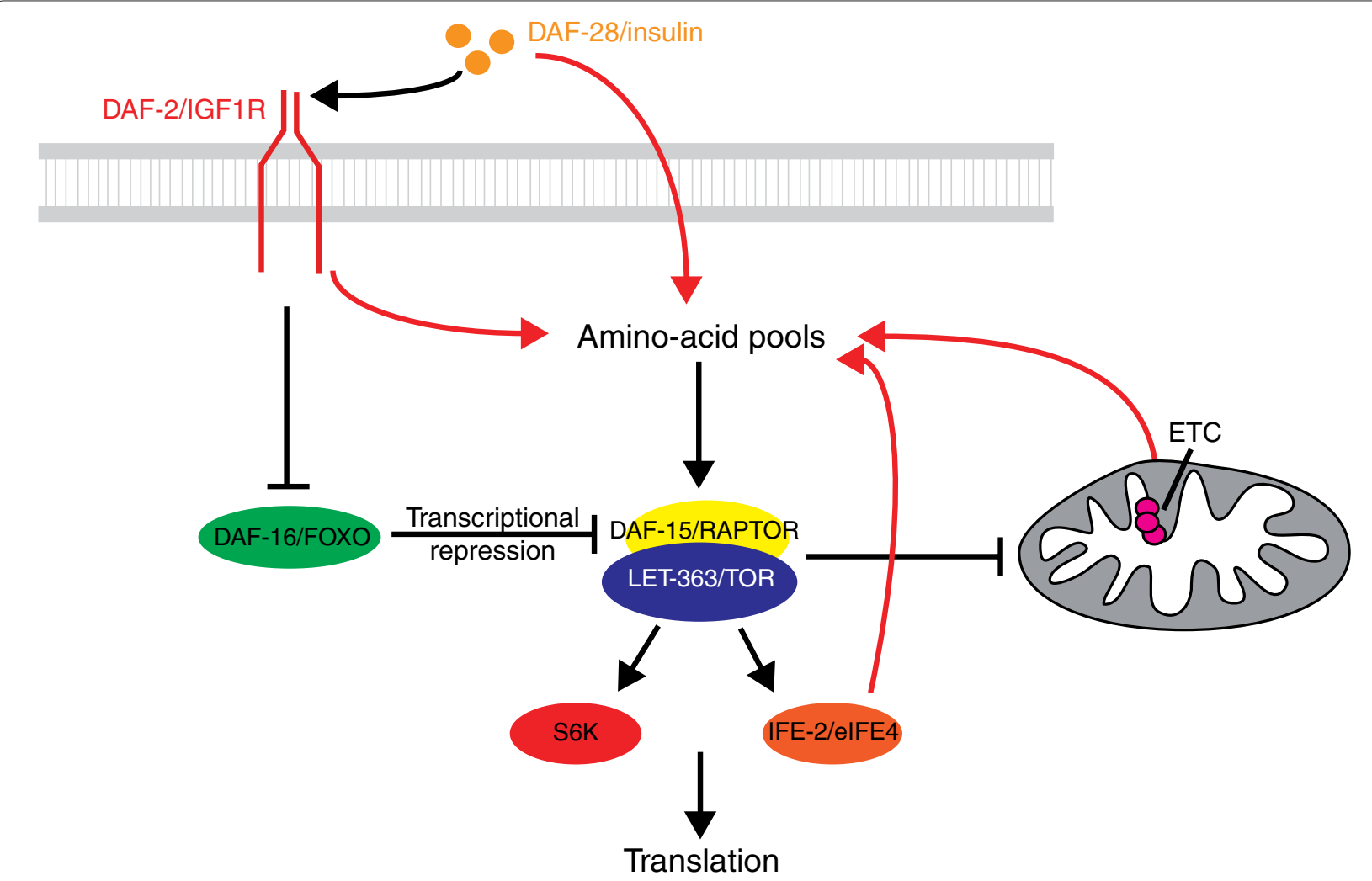

Figure 1. Possible link between accumulation of amino-acid pools, mitochondrial function, and the insulin and TOR pathways in C. elegans longevity. Black lines indicate the activating or repressive function of members of different signal transduction pathways. Amino-acid pools and other nutrients activate TOR signaling, which then results in increased translation following activation of the protein kinase S6K and the translation initiation factor elFE4 and inhibition of mitochondrial biogenesis. However, mutations in DAF-2/IGF1R result in the activation of DAF-16, which represses daf- 15 transcription to reduce TOR signaling. Red arrows indicate the effects of mutations on cellular amino-acid pools. Specifically, Fuchs et al. [7] show that mutations in daf-2/IGF1R, daf-28/insulin and ife-2/elFE4 cause an increase in amino acid levels, especially branched-chain amino acids. Falk et al. [9] showed that mutations affecting complexes I, II and III of the electron transport chain (ETC), including some that increase life span, also result in accumulation of branched-chain amino acids. Mutations that confer longevity through different pathways share the metabolic signature of increased branched-chain amino-acid pools.

the mitochondrial electron transport chain (ETC) complexes I, II and III [9]. At least some of these ETC mutants are long-lived. The increase in branched-chain amino acids in long-lived worms is intriguing for two reasons. First, it opens the possibility that protein metabolism plays an important role in life-span determination. Second, branched-chain amino acids are known to stimulate protein synthesis and inhibit protein degradation in higher eukaryotes [10], a phenomenon mediated by the TOR (target of rapamycin) pathway.

\section{Dietary restriction and metabolism}

Although it might seem reasonable to assume that dietary restriction exerts its life-prolonging effects by reducing metabolic function, recent reports argue to the contrary (reviewed in [11]). Worms on a dietary restriction regimen induced genetically (by eat mutations), or by dilution of nutrients, or by exposure to axenic medium (medium that supports growth without bacteria), actually had increased metabolic rates, as measured by oxygen consumption and heat production. The mechanism by which dietary restriction regulates life span in worms is therefore not clear. While it seems to act by regulating the insulin pathway in Drosophila and rats, the IIS pathway is not responsible for dietary-restriction-induced longevity in C. elegans.

The most likely pathway exploited by dietary restriction in C. elegans is the TOR pathway. The physiological role of TOR kinase is to sense nutrient levels - such as cellular amino-acid pools - and to regulate transcription and protein biogenesis and degradation accordingly. TOR exists in two highly conserved protein complexes: TORC1, which regulates cell growth, protein synthesis and autophagy; and TORC2, which regulates cytoskeletal reorganization [12]. Both complexes regulate the metabolic state of C. elegans. TOR activates the ribosomal p70 S6 kinase (S6K) and the translation initiation factor eIF4E. The latter is encoded by ife- 2 in C. elegans, and a 
mutant in this gene was studied in the metabolomic analysis performed by Fuchs et al. [7]. It was previously observed that knockdown of TOR/let-363 pathway in C. elegans results in an almost twofold increase in life span [13]. Although not mentioned by Fuchs et al. [7], their metabolic profiles fit very well with a model whereby TOR modulates life span via ife-2: downregulation of TOR increases longevity; ife- 2 is an effector of the TOR pathway and animals carrying a mutation in this gene also live longer; and ife-2 mutants accumulate pools of amino acids that are known to induce protein biogenesis and inhibit protein degradation. These data naturally lead to the speculation that the longevity of IIS mutants is at least partially derived from downregulation of TOR.

Mutation of DAF-15/RAPTOR, an activator of TOR, results in increased C. elegans life span [13]. Interestingly, daf- 15 is directly regulated by DAF-16, the ultimate effector of the IIS pathway. It was therefore proposed that mutations reducing IIS signaling (such as daf-2 mutations) activate DAF-16, which then represses daf-15 to decrease the function of TOR and enhance longevity (Figure 1). The increased amino-acid pools found by Fuchs et al. [7] in the IIS mutants daf-2 and daf- 28 could be explained by the consequent downregulation of TOR, which in turn would result in decreased translation and consequent accumulation of amino-acid pools. Mutations in ife-2 would also result in increased amino-acid pools.

\section{Branched-chain amino acids and longevity}

Fuchs et al. [7] may, in fact, hold a clue to one of the mysteries of the aging field: why do translation-defective mutants live longer? If translation mutants such as ife-2 accumulate amino acids, they would mimic the conditions arising in mitochondrial ETC mutants and IIS pathway mutants. TOR pathway mutants would be predicted to have very similar metabolic profiles to ETC, daf-2 or ife- 2 mutants. Analyzing the metabolomes of TOR mutants and translation-defective mutants could therefore shed some light on this problem.

In conclusion, the belief that decreased metabolism leads to longevity is, so far, a generalization that extends beyond the current evidence. We know that genes involved in metabolic control, such as daf-2, regulate life span, but we do not know if overall metabolism is downregulated in these mutants [11]. For instance, daf-2 mutants exhibit decreased carbohydrate metabolism, but gene-expression data suggest that lipid utilization pathways are actually upregulated in these mutants [8].

Life-span-prolonging effects of downregulating protein synthesis might be specific to C. elegans and other invertebrates. The soma of the adult nematode is postmitotic, and metabolic control might have different effects in C. elegans (where adult cell and tissue replacement does not occur) from those in higher eukaryotes, where compromised cells can be eliminated by apoptosis and replaced. C. elegans cells might have a higher tolerance for cellular insults and decreased metabolism. It is conceivable that translation of new proteins and other cell-maintenance processes may be more important to $C$. elegans than to higher organisms, as C. elegans somatic cells cannot be replaced.

Although the role of metabolism in aging is not straightforward, the metabolomics of longevity mutants may provide some answers. It is interesting to notice that several classes of long-lived mutants - mitochondrial ETC mutants, IIS mutants, and translation mutants - all have increased levels of branched-chain amino acids $[7,9]$. Metabolomic profiles of TOR pathway mutants, dietary restriction mutants and other translation mutants could reveal an under-appreciated function of aminoacid metabolism in longevity.

Published: 10 February 2010

\section{References}

1. Morris JZ, Tissenbaum HA, Ruvkun G: A phosphatidylinositol-3-OH kinase family member regulating longevity and diapause in Caenorhabditis elegans. Nature 1996, 382:536-539.

2. Kimura KD, Tissenbaum HA, Liu Y, Ruvkun G: daf-2, an insulin receptor-like gene that regulates longevity and diapause in Caenorhabditis elegans. Science 1997, 277:942-946.

3. Gems D, Sutton AJ, Sundermeyer ML, Albert PS, King KV, Edgley ML, Larsen PL, Riddle DL: Two pleiotropic classes of daf-2 mutation affect larval arrest, adult behavior, reproduction and longevity in Caenorhabditis elegans. Genetics 1998, 150:129-155.

4. Jensen VL, Gallo M, Riddle DL: Targets of DAF-16 involved in Caenorhabditis elegans adult longevity and dauer formation. Exp Gerontol 2006, 41:922-927.

5. Longo VD, Finch CE: Evolutionary medicine: from dwarf model systems to healthy centenarians? Science 2003, 299:1342-1346.

6. Wallace DC: Mitochondrial diseases in man and mouse. Science 1999, 283:1482-1488.

7. Fuchs S, Bundy JG, Davies SK, Viney JM, Swire JS, Leroi AM: A metabolic signature of long life in Caenorhabditis elegans. BMC Biol. 2010, 8:14

8. Halaschek-Wiener J, Khattra JS, McKay S, Pouzyrev A, Stott JM, Yang GS, Holt RA, Jones SJ, Marra MA, Brooks-Wilson AR, Riddle DL: Analysis of long-lived C. elegans daf-2 mutants using serial analysis of gene expression. Genome Res 2005, 15:603-615.

9. Falk M, Zhang Z, Rosenjack J, Nissim I, Daikhin E, Nissim I, Sedensky MM, Yudkoff M, Morgan PG: Metabolic pathway profiling of mitochondrial respiratory chain mutants in C. elegans. Mol Genet Metab 2008, 93:388-397.

10. May ME, Buse MG: Effects of branched-chain amino acids on protein turnover. Diabetes Metab Rev 1989, 5:227-245.

11. Houthoofd $\mathrm{K}$, Johnson TE, Vanfleteren JR: Dietary restriction in the nematode Caenorhabditis elegans. J Gerontol A Biol Sci Med Sci 2005, 60:1125-1131

12. Bhaskar PT, Hay N: The two TORCs and Akt. Dev Cell 2007, 12:487-502

13. Jia K, Chen D, Riddle DL: The TOR pathway interacts with the insulin signaling pathway to regulate C. elegans larval development, metabolism and life span. Development 2004, 131:3897-3906.

doi:10.1186/jbiol215

Cite this article as: Gallo M, Riddle DL: Regulation of metabolism in Caenorhabditis elegans longevity. Journal of Biology 2010, 9:7. 